\title{
Mediterranean diet score and incidence of IHD: a global comparative study
}

\author{
Ayako Sezaki ${ }^{1, *}$, Tomoko Imai ${ }^{1}$, Keiko Miyamoto², Fumiya Kawase ${ }^{2}$ and \\ Hiroshi Shimokata ${ }^{2}$ \\ 'Department of Food Science and Nutrition, Doshisha Women's College of Liberal Arts, Teramachi-Nishi-iru, \\ Imadegawa-dori, Kamigyo-ku, Kyoto 602-0893, Japan: ${ }^{2}$ Graduate School of Nutritional Science, Nagoya \\ University of Arts and Sciences, Aichi, Japan
}

Submitted 30 June 2018: Final revision received 18 0ctober 2018: Accepted 26 November 2018: First published online 12 February 2019

\begin{abstract}
Objective: The aim of the present study was to clarify the global relationship between Mediterranean diet score (MDS) and the incidence of IHD by country using international statistics.

Design: The incidence of IHD by country was derived from the Global Burden of Disease (GBD) database. Average supplies of food (g/d per capita) and energy (kcal/d per capita) by country, excluding loss between production and household, were obtained from the FAOSTAT database. MDS was evaluated based on the total score of nine food items that characterize the Mediterranean diet. The association between MDS and the incidence of IHD was examined in countries with a population of 1 million or greater using a general linear model controlled for socio-economic and lifestyle variables.

Setting: Population data from global international databases.

Participants: One hundred and thirty-two countries with a population of over 1 million.

Results: MDS was inversely correlated with obesity rate, ageing rate, years of education and IHD incidence; however, no associations were found with gross domestic product, life expectancy, smoking rate, energy supply or health expenditure. In the general linear model of IHD incidence by MDS controlled for socio-economic and lifestyle variables, the $\beta$ of the MDS was $-26 \cdot 4$ (sE 8.6; $P<0 \cdot 01)$.

Conclusions: The results of this global international comparative study confirmed that the Mediterranean diet is inversely associated with the incidence of IHD.
\end{abstract}

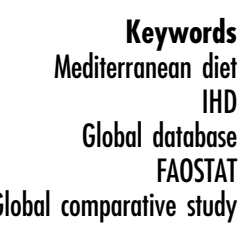

Adherence to the Mediterranean diet, which is believed to be a healthy diet, has been widely associated with good human health. In 2010, the Mediterranean diet was registered on the Representative List of the Intangible Cultural Heritage of Humanity by the United Nations Educational, Scientific and Cultural Organization, as it constitutes 'a set of skills, knowledge, practices and traditions ranging from the landscape to the table, including the crops, harvesting, fishing, conservation, processing, preparation and, particularly, consumption of food ${ }^{,(1)}$.

The traditional Mediterranean diet is characterized by high consumption of fruits and nuts, vegetables, legumes and unprocessed cereals, and low consumption of meat and dairy products. Although alcohol consumption is common in the traditional Mediterranean diet, it is generally in the form of wine, in moderation and, as a rule, only during meals. Total lipid intake can be high (about or in excess of $40 \%$ of total energy intake, as in Greece) or moderate (about $30 \%$ of total energy intake, as in Italy); however, in all instances, the ratio of the beneficial monounsaturated to the non-beneficial saturated lipids is high, owing to the high MUFA content of olive oil, which is used liberally. Finally, because of its proximity to the ocean, moderate amounts of fish can be consumed ${ }^{(2)}$.

The traditional Mediterranean dietary pattern was reported by Keys and colleagues in the Seven Countries Study ${ }^{(3)}$. An important conclusion of their study, based largely on ecological evidence, was that the low saturated lipid content of the Mediterranean diet could explain the low incidence of CHD in Mediterranean countries.

One of the reasons why epidemiological studies on the Mediterranean diet have been conducted worldwide was the development of the Mediterranean diet score (MDS). The Mediterranean diet was conceptualized in the 1960s to 
reflect the meals that were typically eaten in the coastal areas of the Mediterranean (Southern Italy, Greece, especially in Crete, etc. $)^{(4)}$. It is essentially a frugal diet that was traditionally followed by poor rural societies ${ }^{(5)}$. Among a variety of versions, the MDS created by Trichopoulou et al. in 1995 and updated thereafter ${ }^{(2)}$ is used most widely ${ }^{(6,7)}$. A gradient of adherence to the traditional Greek Mediterranean diet was constructed from nine nutritional components (fruits and nuts; vegetables; legumes; cereals; fish; olive oil; meat and poultry; dairy; wine), with values of 0 or 1 assigned to each component using the medians as cut-off values.

In a recent systematic review and meta-analysis, Grosso et $a l .{ }^{(8)}$ reported finding an association between adherence to the Mediterranean diet and lower incidence of and mortality from CVD, including CHD, myocardial infarction and stroke. The most protective effects were associated with increased consumption of olive oil, fruits, vegetables, legumes and nuts, and moderate intake of wine (especially red wine).

However, the relationship between MDS and the incidence of IHD has yet to be elucidated in any global international comparative studies. Therefore, the purpose of the present study was to clarify the relationship between MDS and the incidence of IHD using population data from global international databases.

\section{Methods}

\section{Variables}

Incidence of IHD

The Global Burden of Disease (GBD) database is the most comprehensive source of comparable summary population health measures because of its inclusion of country-level results, uncertainty quantification and effort to maximize comparability across space, time and different health conditions ${ }^{(9)}$. For the purposes of the present study, we used the age-standardized incidence of IHD per 100000 people in 2010 .

\section{Food supply}

Covering all regional groupings of the FAO from 1961 to the most recent year available, the FAOSTAT database provides food and agricultural data for more than 245 countries and territories ${ }^{(10)}$. We examined the most recent data (since 2010) using 129 animal and vegetable food supply items after excluding the duplicates.

\section{Mediterranean diet score}

MDS were calculated according to the most commonly used index by Trichopoulou et $a l^{(2)}$. Items corresponding to the nine food components that comprise the MDS (vegetables; legumes; fruits and nuts; cereals; fish; olive oil; meat and meat products; dairy products; wine) were extracted. Each component was calculated as the daily supply per capita ( $\mathrm{g} / \mathrm{d}$ per capita). In the present study, the amount of olive oil consumed, rather than the ratio of monounsaturated to saturated lipids, was used. The amount of ethanol from wine was used as the amount of wine intake. The presumed beneficial components (vegetables, legumes, fruits and nuts, cereals, fish and olive oil) below the medians of the countries were assigned a value of 0 , and 1 otherwise. By contrast, the presumed detrimental components (meat and dairy) below the medians were assigned a value of 1 , and 0 otherwise. To calculate alcohol intake, we modified the MDS based on $50 \mathrm{~g}$ of ethanol as a standard according to median alcohol intake values. The amount of wine consumed (as ethanol) was rated as 1 for over $50 \mathrm{~g}$, and 0 otherwise. Calculations were made for supply amount per $4184 \mathrm{~kJ}$ (1000 kcal), except for wine. Therefore, a 10-point MDS that could take a value from 0 (minimal adherence) to 9 (maximal adherence) was constructed.

\section{Covariables}

Food energy supply (kcal/d per capita) was determined based on the FAOSTAT database. Gross domestic product (GDP) per capita (\$US/capita) and years of education were identified using data from the United Nations Educational, Scientific and Cultural Organization Institute for Statistics' database ${ }^{(11)}$. Current smoking rates were identified using data from the GBD database ${ }^{(9)}$. The prevalence of obesity (BMI $\geq 30 \cdot 0 \mathrm{~kg} / \mathrm{m}^{2}$ ) was calculated based on the WHO database ${ }^{(12)}$. The percentage of the population over 65 years of age (ageing rate) and health expenditures (\$US/capita) were identified using the World Bank database $^{(13)}$. The most recent data available (since 2010) in all databases were used.

\section{Statistical analysis}

Analysis was conducted for the 132 countries with a population of over 1 million and no missing data. Each country was divided into two groups based on the MDS low-score countries ( $\leq$ median) and high-score countries ( $>$ median) - and then food supply amounts and socioeconomic and lifestyle variables were compared between the groups using the $t$ test. The interrelationships among these variables were assessed by single and partial correlation analyses controlled for covariables.

The effects of MDS on the incidence of IHD were evaluated using three general linear models. Model 1 was a single regression model of MDS $v$, the incidence of IHD; Model 2 was a multiple regression model controlled for GDP per capita; and Model 3 was a multiple regression model further controlled for socio-economic and lifestyle variables. Akaike's information criterion was used as an estimator of the relative quality of models and the Bayesian information criterion was used as a criterion for model selection. To eliminate multiple collinearity, the variance 
inflation factor was calculated and variables were chosen so that the variance inflation factor of all variables was 10 or less. R version 3.3.3 $3^{(14)}$ was used for all analyses and values of $P<0.05$ were taken to indicate statistical significance.

\section{Results}

MDS were distributed from 1 to 8 points and were not invariably high in the Mediterranean countries. MDS were generally higher in Asian and African countries, and generally lower in South American, Northern European, Eastern European and former Soviet bloc countries (Fig. 1).

The characteristics of countries with high and low MDS are shown in Table 1 . The median score was 3. The incidence of IHD was significantly lower in countries with a high than in countries with a low MDS $(P=0 \cdot 014)$. In countries with higher scores, the supplies of fruits and nuts, vegetables and legumes were significantly higher, and those of dairy products and meat were significantly lower, compared with countries with lower scores. No significant differences were seen in cereals, fish, olive oil or wine. Regarding socio-economic variables, countries with higher MDS had lower ageing rates and fewer years of education compared with countries with lower scores. Regarding lifestyle variables, countries with higher scores had lower smoking rates.

Table 2 shows the single and partial correlation coefficients controlled for covariables. The results of single correlation analysis showed that the incidence of IHD was inversely correlated with GDP, life expectancy and health expenditure. MDS were inversely correlated with ageing rate, education, obesity rate and $\mathrm{IHD}$, but were not related to GDP, life expectancy, health expenditure, smoking rate or energy supply. The single correlation coefficient between MDS and the incidence of IHD was -0.245 $(P<0 \cdot 01)$.
The results of partial correlation analysis controlled for covariables showed that the incidence of IHD was inversely correlated with GDP and life expectancy, and positively correlated with education, smoking rate and energy supply. MDS were inversely correlated with obesity rate and IHD. The partial correlation coefficient between MDS and the incidence of IHD was $-0.209(P<0.05)$.

The relationship between MDS and the incidence of IHD is shown in a bubble chart in Fig. 2, with bubble size representing the GDP per capita. The score in Japan was 5, the same as those for Italy, Spain and Greece, which were all higher than the score of 4 points in France. The single regression line between MDS and the incidence of IHD was $y=-26 \cdot 1 x+482 \cdot 9$.

Table 3 shows the results of the three general linear models for the incidence of IHD. In Model 1, the single regression coefficient $(\beta)$ of the MDS was $-26 \cdot 1$ (SE 9.1; $P<0.001$ ). In Model 2, the partial regression coefficient controlled for GDP per capita was -28.5 (se $8 \cdot 3 ; P<0 \cdot 001$ ). In Model 3, the partial regression coefficient of the MDS controlled for GDP, ageing rate, education, smoking rate, energy supply and obesity rate was also significant ( $-26 \cdot 4$ (se 8.6), $P<0 \cdot 01$ ). In the model in which both GDP and health expenditure were included, the variance inflation factor of GDP and health expenditure was $18 \cdot 2$ and 16.7, respectively. Therefore, health expenditure was excluded from the covariables in Model 3. Akaike's information criterion was lowest in Model 3 and the Bayesian information criterion was lowest in Model 2.

\section{Discussion}

Using population data from global international databases, the present study provides the first evidence of a significant relationship between the Mediterranean diet and the incidence of IHD. This association remained after controlling for socio-economic and lifestyle variables.

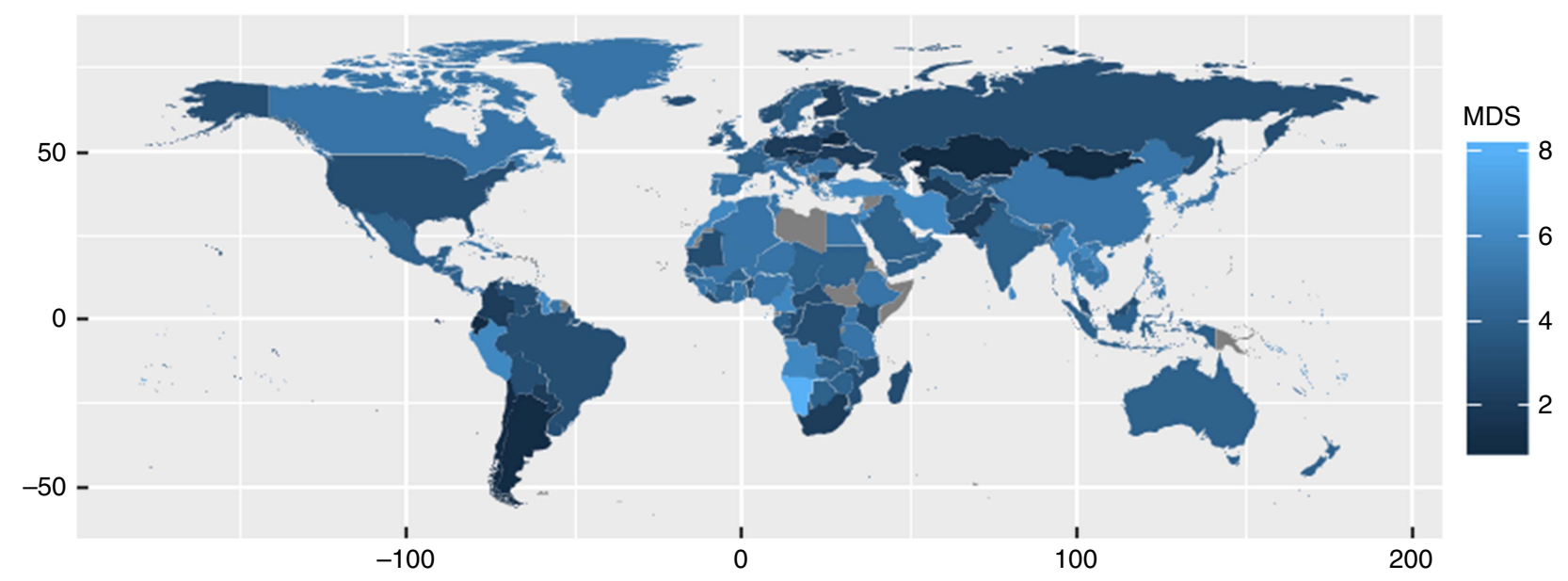

Fig. 1 Global distribution of Mediterranean diet score (MDS) by country. For the countries shown in grey, no data on food supply after 2010 were obtained 
Table 1 Characteristics of countries with low and high Mediterranean diet score (MDS) in global international databases

\begin{tabular}{|c|c|c|c|c|c|}
\hline & \multicolumn{2}{|c|}{$\begin{array}{l}\text { Low-score countries } \\
\text { (MDS } \leq \text { mediant; } n \text { 46) }\end{array}$} & \multicolumn{2}{|c|}{$\begin{array}{l}\text { High-score countries } \\
(\text { MDS }>\text { mediant, } n \text { 86) }\end{array}$} & \multirow[b]{2}{*}{$P$ value $\ddagger$} \\
\hline & Mean & SD & Mean & SD & \\
\hline MDS & $2 \cdot 41$ & 0.75 & 4.72 & 0.78 & $<0.001$ \\
\hline IHD incidence (per 100000 people) & $422 \cdot 2$ & $164 \cdot 3$ & $358 \cdot 3$ & $126 \cdot 3$ & 0.014 \\
\hline \multicolumn{6}{|l|}{ Food supply } \\
\hline Fruits and nuts $(\mathrm{g} / \mathrm{d})$ & $210 \cdot 5$ & $96 \cdot 7$ & 259.8 & $150 \cdot 1$ & 0.046 \\
\hline Vegetables $(\mathrm{g} / \mathrm{d})$ & $215 \cdot 4$ & 109.5 & $310 \cdot 0$ & $194 \cdot 1$ & 0.003 \\
\hline Legumes $(\mathrm{g} / \mathrm{d})$ & $17 \cdot 7$ & 11.9 & 33.9 & $21 \cdot 8$ & $<0.001$ \\
\hline Cereals $(g / d)$ & $346 \cdot 7$ & $85 \cdot 3$ & $380 \cdot 6$ & 112.5 & 0.076 \\
\hline Fish $(g / d)$ & $37 \cdot 0$ & 35.4 & $45 \cdot 8$ & $36 \cdot 8$ & 0.183 \\
\hline Olive oil $(\mathrm{g} / \mathrm{d})$ & 0.87 & 1.23 & 2.49 & $6 \cdot 21$ & 0.085 \\
\hline Dairy $(g / d)$ & $429 \cdot 3$ & $274 \cdot 1$ & $282 \cdot 2$ & $252 \cdot 1$ & 0.002 \\
\hline Meat $(\mathrm{g} / \mathrm{d})$ & $168 \cdot 6$ & 76.4 & $121 \cdot 7$ & $80 \cdot 4$ & 0.001 \\
\hline Wine $(g / d)$ & $21 \cdot 6$ & 22.5 & $14 \cdot 2$ & $27 \cdot 2$ & 0.118 \\
\hline \multicolumn{6}{|l|}{ Socio-economic variables } \\
\hline Population (million) & 33.4 & $62 \cdot 6$ & $62 \cdot 63$ & $203 \cdot 2$ & 0.344 \\
\hline GDP (1000 \$US/capita) & 13.5 & $16 \cdot 5$ & $11 \cdot 8$ & $16 \cdot 5$ & 0.574 \\
\hline Ageing rate (\%) & $10 \cdot 8$ & $6 \cdot 3$ & 8.03 & $6 \cdot 16$ & 0.017 \\
\hline Health expenditure (1000 \$US/capita) & 1.37 & $2 \cdot 16$ & $1 \cdot 12$ & 1.96 & 0.505 \\
\hline Education (years) & 9.31 & 3.08 & $7 \cdot 60$ & 3.15 & 0.003 \\
\hline Life expectancy (years) & $72 \cdot 3$ & 8.2 & $72 \cdot 1$ & $8 \cdot 1$ & 0.886 \\
\hline \multicolumn{6}{|l|}{ Lifestyle variables } \\
\hline Smoking (\%) & 19.5 & $7 \cdot 8$ & $16 \cdot 2$ & $7 \cdot 7$ & 0.021 \\
\hline Energy supply $(\mathrm{kJ} / \mathrm{d})$ & $12113 \cdot 5$ & $2028 \cdot 0$ & $11849 \cdot 9$ & 1924.6 & 0.463 \\
\hline Energy supply (kcal/d) & $2895 \cdot 2$ & 484.7 & $2832 \cdot 2$ & $460 \cdot 0$ & 0.463 \\
\hline Obesity (\%) & $19 \cdot 1$ & $7 \cdot 7$ & $16 \cdot 5$ & $9 \cdot 8$ & 0.13 \\
\hline
\end{tabular}

GDP, gross domestic product.

Analysis was conducted for the 132 countries with a population of over 1 million without missing data. Each country was divided into two groups based on MDS. Food supply and socio-economic and lifestyle variables were compared between the two groups using the $t$ test.

†The median MDS was 3. A score of 0 indicates minimum adherence and a score of 9 indicates maximum adherence.

‡Student's $t$ test.

The Mediterranean diet, which is regarded as one of the world's healthiest, has been followed and protected by residents of Mediterranean countries for a long time. Numerous studies have been conducted on the Mediterranean diet, including that by Trichopoulou et al. ${ }^{(2)}$.

The health benefits of the Mediterranean diet have been widely researched in the past, particularly in terms of the incidence of $\mathrm{CVD}^{(15-18)}$ and the prevention of type 2 diabetes $^{(19,20)}$ and metabolic syndrome ${ }^{(21)}$. The European Prospective Investigation into Cancer and Nutrition (EPIC) study has also reported some benefits of the Mediterranean diet against the occurrence of cancer. Evidence of the potential protection provided by the Mediterranean diet seems stronger for gastric, colorectal and breast cancers, especially when alcohol is excluded from the definition ${ }^{(22,23)}$. In addition, many systematic reviews and meta-analyses have reported the beneficial effects of the Mediterranean diet, and the reliability of these results is considered high ${ }^{(24,25)}$. However, we could not find any studies that analysed a large number of countries simultaneously using the same scoring method. According to the results of the present study, MDS are high in countries in Asia as well as those in the Mediterranean region.
According to the GDB database, the annual global incidence of IHD in 2010 was estimated to be 379.7 per 100000 persons $^{(9)}$. From this value and the results of the present study, the incidence of IHD is expected to decrease by $7 \cdot 1 \%$ for every 1 -point increase in MDS. This suggests the importance of maintaining a healthy diet based on meals rich in fish, vegetables, fruits and cereals.

Scientific evidence has shown that the adoption of the Mediterranean diet is a protective factor against the onset of various types of CVD, cancer, ageing and obesity. As shown by the EPIC study, the Mediterranean diet is most effective for the prevention of several chronic diseases, including cancer. The anti-tumour effects of the Mediterranean diet owe mostly to the combination of antioxidants, fibre and polyunsaturated fats. This dietary pattern is therefore essential as a preventive measure against all-cause mortality ${ }^{(26,27)}$.

In the present study, the intakes of wine, olive oil and grains were not necessarily high in the countries with high MDS. The reason for this may be because of differences in eating habits by region ${ }^{(28)}$. For example, wine and olive oil are consumed on a daily basis in a limited number of countries; in traditional dishes in Asian countries, these foods are rarely used. 


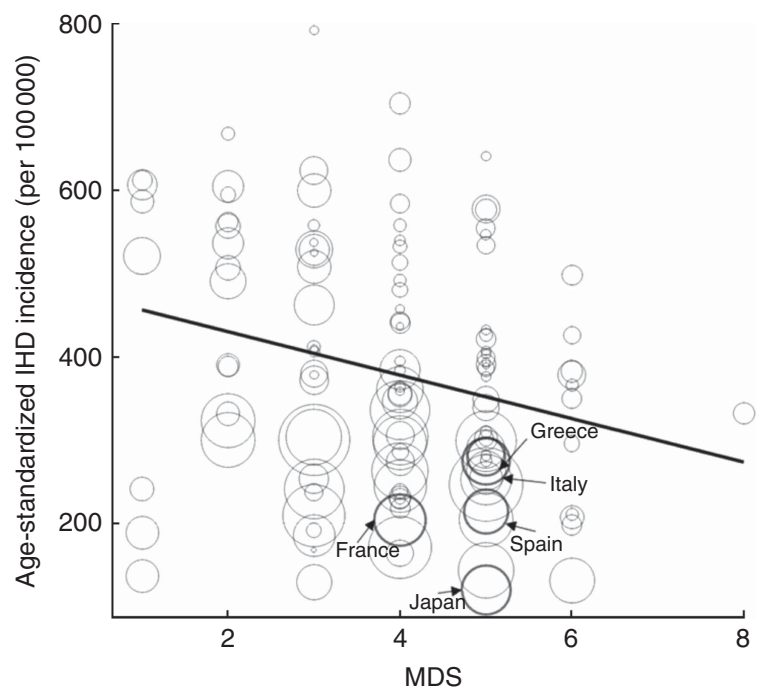

Fig. 2 Mediterranean diet score (MDS) and age-standardized incidence of IHD in the 132 countries with a population of over 1 million in global international databases. Bubble size represents the gross domestic product per capita of each country; represents the regression line $(y=-26 \cdot 1 x+482 \cdot 9)$

The results of such an analysis could be expected to vary depending on the scoring method. For example, our research results have issues, such as including both red and white wine. For more detailed and accurate analyses, it may be necessary to develop a new scoring method focusing on 'groups of foods susceptible to food culture'. If food intake is replaced with nutrient intake, it may be possible to adjust food differences in accordance with differences in food culture and to propose dietary contents to be ingested for each area of the world. Some recent papers have weighted the Mediterranean diet on a nutrient basis ${ }^{(29,30)}$ or created nutrient scores ${ }^{(31-33)}$. Furthermore, a variety of MDS, such as the alternative and relative MDS ${ }^{(34)}$ and Mediterranean Adequacy Index ${ }^{(35)}$, have been developed; however, evidence of the validity of these scores remains insufficient.

In the present study, we used the score created by Trichopoulou et $a l^{(2)}$. We initially included fruits in the score, but fruit consumption is associated with higher socio-economic status, which, in turn, is associated with a comparatively higher consumption of meat ${ }^{(36)}$; this could pose some problems regarding the inclusion of fruits as a variable in the score calculation. Therefore, we conducted an additional analysis excluding fruits. However, no relationship was found between MDS and the incidence of IHD in the correlation or regression analyses. This seems to indicate that fruits play an important role in preventing IHD. Additionally, a previous study reported that higher consumption of fruits is associated with a lower risk of allcause mortality, particularly cardiovascular mortality ${ }^{(36)}$. Consequently, we used Trichopoulou's score, including fruits as a variable.

To calculate alcohol intake, we modified the MDS based on $50 \mathrm{~g}$ of ethanol as a standard based on median alcohol 
Table 3 Partial regression coefficients of Mediterranean diet score (MDS) and covariables in three general linear models for the incidence of IHD in 132 countries with a population of over 1 million in global international databases

\begin{tabular}{|c|c|c|c|c|c|c|}
\hline & \multicolumn{2}{|c|}{ Model 1} & \multicolumn{2}{|c|}{ Model 2} & \multicolumn{2}{|c|}{ Model 3} \\
\hline & $\beta$ & SE & $\beta$ & SE & $\beta$ & SE \\
\hline Intercept & 482.923 & $37.581^{\star * *}$ & 536.962 & $35.605^{\star \star \star}$ & 305.795 & $98 \cdot 936^{\star \star \star}$ \\
\hline MDS & $-26 \cdot 140$ & $9 \cdot 080^{\star \star *}$ & -28.527 & $8 \cdot 261^{* \star *}$ & $-26 \cdot 371$ & $8 \cdot 627^{\star \star}$ \\
\hline GDP & & & -3.602 & $0.674^{\star * *}$ & -4.748 & $1.039^{\star *}$ \\
\hline Ageing rate & & & & & $-3 \cdot 638$ & $3 \cdot 106$ \\
\hline Education & & & & & 11.085 & $6 \cdot 616$ \\
\hline Smoking & & & & & $2 \cdot 726$ & 1.973 \\
\hline Energy & & & & & 0.069 & 0.043 \\
\hline Obesity & & & & & $-3 \cdot 706$ & 1.880 \\
\hline AIC & \multicolumn{2}{|c|}{$1682 \cdot 3$} & \multicolumn{2}{|c|}{1657.9} & \multicolumn{2}{|c|}{1654.7} \\
\hline $\mathrm{BIC}$ & \multicolumn{2}{|c|}{1690.9} & \multicolumn{2}{|c|}{$1669 \cdot 4$} & \multicolumn{2}{|c|}{$1680 \cdot 6$} \\
\hline
\end{tabular}

GDP, gross domestic product; AIC, Akaike's information criterion; BIC, Bayesian information criterion. ${ }^{\star *} P<0.01,{ }^{\star \star \star} P<0.001$.

intake values. There are ongoing discussions about the effect of ethanol intake, but considering past research about the cardiovascular protective effects of ethanol, ethanol intake was used in the score calculations ${ }^{(38,39)}$.

We also performed a sensitivity analysis on the impact of MDS on IHD. In the analysis, the annual incidence rate per 100000 cases of IHD was estimated to be 457.5 at the lowest MDS value and 272.9 at the highest (full adjustment model). The sensitivity analysis between each food group and MDS showed that legumes had the greatest influence on MDS, followed by meat, dairy products, fish, fruits, vegetables and cereals. Olive oil and wine were found to have only a slight influence, likely owing to the fact, from a global point of view, that intake of olive oil and wine is extremely low for most countries.

The present study has several limitations. First, the results consist of country data, not personal data, and it was not possible to analyse by sex or age group. Second, MDS were not calculated based on the actual consumption of foods. The amount of waste at home was considered, but because the disposal rate did not differ greatly depending on the food, the effects of the difference between supply and consumption amounts per $4184 \mathrm{~kJ}$ (1000 kcal) would be not large. Physical activity is an important factor in relation to the incidence of IHD. However, it is virtually impossible to conduct accurate and objective dietary surveys simultaneously around the entire world. In the present study, we used objective data obtained from almost all the countries in the world for the analysis; however, we could not obtain data on physical activity. We obtained inactivity data from the WHO database $^{(40)}$. Although these data, which were obtained from citizens in 108 countries, were incomplete, the association between MDS and the incidence of IHD in the regression model controlled for inactivity, in addition to GDP, ageing rate, years of education, smoking and obesity, was still significant $(\beta=-20 \cdot 3$ (se 9.2); $P<0 \cdot 05$ ).

Several studies have investigated the relationship between the Mediterranean diet and the incidence of IHD by summarizing data from cohort studies in several countries and regions; however, no global-scale studies have been conducted. Similar to previous studies, the results of the present study using population data from global international databases suggest that the Mediterranean diet may be effective for the prevention of IHD. In fact, following the Mediterranean diet may have greater preventive effects on IHD than other lifestyle variables such as smoking, obesity and exercise.

\section{Acknowledgements}

Financial support: This research received no specific grant from any funding agency in the public, commercial or notfor-profit sectors. Conflict of interest: The authors have no conflicts of interest to disclose. Authorship: A.S., T.I., K.M., F.K. and H.S. formulated the research question. A.S. and H.S. designed the study. A.S. carried out the study. A.S., F.K. and H.S. analysed the data. A.S. and H.S. wrote the article. Ethics of human subject participation: Not applicable.

\section{References}

1. United Nations Educational, Scientific and Cultural Organization (2013) Culture I Intangible Cultural Heritage I Lists | Mediterranean diet. https://ich.unesco.org/en/Rl/medi terranean-diet-00884 (accessed December 2017).

2. Trichopoulou A, Costacou T, Bamia C et al. (2003) Adherence to a Mediterranean diet and survival in a Greek population. N Engl J Med 348, 2599-2608.

3. Keys A, Arvanis C \& Blackburn H (1980) Seven Countries: A Multivariate Analysis of Death and Coronary Heart Disease. Cambridge, MA: Harvard University Press.

4. Willett WC, Sacks F, Trichopoulou A et al. (1995) Mediterranean diet pyramid: a cultural model for healthy eating. Am J Clin Nutr 61, 6 Suppl., 1402S-1406S.

5. Bach-Faig A, Berry EM, Lairon D et al:; Mediterranean Diet Foundation Expert Group (2011) Mediterranean diet pyramid today. Science and cultural updates. Public Health Nutr 14, 2274-2284. 
6. Trichopoulou A, Kouris-Blazos A, Wahlqvist ML et al. (1995) Diet and overall survival in elderly people. BMJ 311, 1457-1460.

7. Bach A, Serra-Majem L, Carrasco JL et al. (2006) The use of indexes evaluating the adherence to the Mediterranean diet in epidemiological studies: a review. Public Health Nutr 9, 132-146.

8. Grosso G, Marventano S, Yang J et al. (2015) A comprehensive meta-analysis on evidence of Mediterranean diet and cardiovascular disease: are individual components equal? Am J Clin Nutr 101, 783-793.

9. GBD 2015 DALYs and HALE Collaborators (2016) Global, regional, and national disability-adjusted life-years (DALYs) for 315 diseases and injuries and healthy life expectancy (HALE), 1990-2015: a systematic analysis for the Global Burden of Disease Study 2015. Lancet 388, 1603-1658.

10. Food and Agricultural Organization of the United Nations (2017) FAOSTAT database. http://www.fao.org/faostat/ (accessed December 2017).

11. United Nations Educational, Scientific and Cultural Organization, Institute for Statistics (2016) UIS.Stat. http://data.uis. unesco.org/ (accessed December 2017).

12. World Health Organization (2016) Global Health Observatory data repository - Body Mass Index (BMI). http://apps. who.int/gho/data/node.main.BMIANTHROPOMETRY?lang=en (accessed December 2017).

13. The World Bank (2016) World Bank Open Data. https:// data.worldbank.org (accessed December 2017).

14. R Core Team (2017) R: A Language and Environment for Statistical Computing. Vienna: R Foundation for Statistical Computing; available at https://www.R-project.org/

15. Estruch R, Ros E, Salas-Salvado J et al.; PREDIMED Study Investigators (2013) Primary prevention of cardiovascular disease with a Mediterranean diet. N Engl J Med 368, 1279-1290.

16. de Lorgeril M, Renaud S, Mamelle N et al. (1994) Mediterranean $\alpha$-linolenic acid-rich diet in secondary prevention of coronary heart disease. Lancet 343, 1454-1459.

17. de Lorgeril M (2013) Mediterranean diet and cardiovascular disease: historical perspective and latest evidence. Curr Atheroscler Rep 15, 370.

18. Martinez-Gonzalez MA \& Bes-Rastrollo M (2014) Dietary patterns, Mediterranean diet, and cardiovascular disease. Curr Opin Lipidol 25, 20-26.

19. Martinez-Gonzalez MA, de la Fuente-Arrillaga C, NunezCordoba JM et al. (2008) Adherence to Mediterranean diet and risk of developing diabetes: prospective cohort study. BMJ 336, 1348-1351.

20. Salas-Salvado J, Bullo M, Estruch R et al. (2014) Prevention of diabetes with Mediterranean diets: a subgroup analysis of a randomized trial. Ann Intern Med 160, 1-10.

21. Kastorini CM, Milionis HJ, Esposito K et al. (2011) The effect of Mediterranean diet on metabolic syndrome and its components: a meta-analysis of 50 studies and 534,906 individuals. J Am Coll Cardiol 57, 1299-1313.

22. Couto E, Boffetta P, Lagiou P et al. (2011) Mediterranean dietary pattern and cancer risk in the EPIC cohort. $B r J$ Cancer 104, 1493-1499.

23. Buckland G, Travier N, Cottet V et al. (2013) Adherence to the Mediterranean diet and risk of breast cancer in the European prospective investigation into cancer and nutrition cohort study. Int J Cancer 132, 2918-2927.

24. Sofi F, Abbate R, Gensini GF et al. (2010) Accruing evidence on benefits of adherence to the Mediterranean diet on health: an updated systematic review and meta-analysis. Am J Clin Nutr 92, 1189-1196.

25. Daniele ND, Noce A, Vidiri MF et al. (2017) Impact of Mediterranean diet on metabolic syndrome, cancer and longevity. Oncotarget 8, 8947-8979.

26. Menotti A, Kromhout D, Puddu PE et al. (2017) Baseline fatty acids, food groups, a diet score and 50-year all-cause mortality rates. An ecological analysis of the Seven Countries Study. Ann Med 49, 718-727.

27. Martínez-González MA \& Sánchez-Villegas A (2004) The emerging role of Mediterranean diets in cardiovascular epidemiology: monounsaturated fats, olive oil, red wine or the whole pattern? Eur J Epidemiol 19, 9-13.

28. Davis C, Bryan J, Hodgson J et al. (2015) Definition of the Mediterranean diet: a literature review. Nutrients 7, 9139-9153.

29. de Lorgeril M, Salen P, Martin JL et al. (1999) Mediterranean diet, traditional risk factors, and the rate of cardiovascular complications after myocardial infarction: final report of the Lyon Diet Heart Study. Circulation 99, 779-785.

30. Kafatos A, Verhagen H, Moschandreas J et al. (2000) Mediterranean diet of Crete: foods and nutrient content. $J \mathrm{Am}$ Diet Assoc 100, 1487-1493.

31. Buckland G, González CA, Agudo A et al. (2009) Adherence to the Mediterranean diet and risk of coronary heart disease in the Spanish EPIC Cohort Study. Am J Epidemiol 170, 1518-1529.

32. Itsiopoulos C, Brazionis L, Kaimakamis M et al. (2011) Can the Mediterranean diet lower HbA1c in type 2 diabetes? Results from a randomized cross-over study. Nutr Metab Cardiovasc Dis 21, 740-747.

33. Guallar-Castillon $\mathrm{P}$, Rodriguez-Artalejo $\mathrm{F}$, Tormo $\mathrm{M}$ et al. (2012) Major dietary patterns and risk of coronary heart disease in middle-aged persons from a Mediterranean country: the EPIC-Spain cohort study. Nutr Metab Cardiovasc Dis 22, 192-199.

34. Fung TT, Rexrode KM, Mantzoros CS et al. (2009) Mediterranean diet and incidence of and mortality from coronary heart disease and stroke in women. Circulation 119, 1093-1100.

35. Fidanza AA \& Fidanza F (2004) Mediterranean Adequacy Index of Italian diets. Public Health Nutr 7, 937-941.

36. Dubowitz T, Heron M, Bird CE et al. (2008) Neighborhood socioeconomic status and fruit and vegetable intake among Whites, Blacks, and Mexican-Americans in the United States. Am J Clin Nutr 87, 1883-1891.

37. Wang X, Ouyang Y \& Liu J (2014) Fruit and vegetable consumption and mortality from all causes, cardiovascular disease, and cancer: systematic review and dose-response meta-analysis of prospective cohort studies. BMJ $\mathbf{3 4 9}$, g5472.

38. Chiva-Blanch G, Arranz S, Lamuela-Raventos RM et al. (2013) Effects of wine, alcohol and polyphenols on cardiovascular disease risk factors: evidences from human studies. Alcohol Alcohol 48, 270-277.

39. Roerecke M \& Rehm J (2012) The cardioprotective association of average alcohol consumption and ischaemic heart disease: a systematic review and meta-analysis. Addiction 107, 1246-1260.

40. World Health Organization (2016) Global Health Observatory visualizations I Indicator Metadata Registry I Prevalence of insufficient physical activity among adults aged $18+$ years. http://apps.who.int/gho/data/node.wrapper.imr?xid=2381 (accessed December 2017). 